\title{
Current-in-plane Tunneling Measurement through Patterned Contacts on Top Surfaces of Magnetic Tunnel Junctions
}

\author{
Ching-Ming Lee ${ }^{1,2}$, Lin-Xiu Ye ${ }^{1}$, Jia-Mou Lee ${ }^{1,3}$, Yu-Cyun Lin², Chao-Yuan Huang ${ }^{1,4}$, \\ J. C. $\mathrm{Wu}^{5}$, Masakiyo Tsunoda ${ }^{6}$, Migaku Takahashi ${ }^{6}$, and Te-ho $\mathrm{Wu}^{1,2,7 *}$ \\ ${ }^{1}$ Taiwan SPIN Research Center, National Yunlin University of Science and Technology, Douliou 64002, Taiwan \\ ${ }^{2}$ Graduate School of Materials Science, National Yunlin University of Science and Technology, Douliou 64002, Taiwan \\ ${ }^{3}$ Graduate School of Engineering Science and Technology, National Yunlin University of Science and Technology, Douliou 64002, Taiwan \\ ${ }^{4}$ Department of Physics, National Taiwan Normal University, Taipei 10610, Taiwan \\ ${ }^{5}$ Taiwan SPIN Research Center, National Changhua University of Education, Changhua 500, Taiwan \\ ${ }^{6}$ Department of Electronic Engineering, Tohoku University, Sendai, Japan \\ 7 Graduate School of Information Technology, Overseas Chinese University, Taichung 407, Taiwan
}

(Received 20 April 2011, Received in final form 10 May 2011, Accepted 12 May 2011)

\begin{abstract}
This study reports an alternative method for measuring the magnetoresistance of unpatterned magnetic tunnel junctions similar to the current-in-plane tunneling (CIPT) method. Instead of using microprobes, a series of point contacts with different spacings are coated on the top surface of the junctions and R-H loops at various spacings are then measured by the usual four-point probe method. The values of magnetoresistance and resistance-area products can be obtained by fitting the measured data to the CIPT theoretical model. The test results of two types of junctions were highly similar to those obtained from standard CIPT tools. The proposed method may help to accelerate the process for evaluating the quality of magnetic tunnel junctions when commercial CIPT tools are not accessible.
\end{abstract}

Keywords : magnetic tunnel junctions, current-in-plane tunneling, magnetoresistance, resistance-area product

\section{Introduction}

The development of magnetic tunneling junctions (MTJs) mainly concentrates on raising magnetoresistance ratios (MR) while reducing the values of the resistance-area product (RA) [1,2]. To measure MR and RA values, the blanket wafers must usually be patterned into devices. However, the involved lithographic process is expensive and time-consuming because it is a multi-step process. Accelerating the development period is critical for assessing the quality of entire blanket wafers before patterning. A newly proposed current-in-plane tunneling (CIPT) method $[3,4]$ offers a rapid and nondestructive technique for measuring both MR and RA values simultaneously. Unfortunately, the commercial equipment is expensive and dedicated. Furthermore, the microprobe tips are easily worn out and vulnerable to rough film surfaces. In the present study, we propose a compromised method that replaces

\footnotetext{
*Corresponding author: Tel: +886-5-5342601\#3166

Fax: +886-5-5312174, e-mail: wuth@yuntech.edu.tw
}

the expensive 12-point microprobes with a series of point contacts of different spacings deposited onto the MTJ's top surface. Each contact connects to a probe pad through a sensing lead. The conventional four-point probe method can then be applied to the selected pads for R-H loops measurements. Compared to the process of etching through the whole MTJ stack to manufacture submicron-sized devices, fabrication of these top contacts involves fewer microfabrication steps and, thus, can accelerate the process of quality evaluation. In addition, measuring MR under various field directions and temperatures is more flexible using conventional four-point probe platforms. In particular, the proposed method can be directly applied to perpendicular magnetic tunnel junctions (pMTJs).

\section{Experimental Details}

The first sample MTJ thin film consisting of $\mathrm{SiO}_{2} /$ $\mathrm{Ta}(5) / \mathrm{Ru}(40) / \mathrm{Ta}(5) / \mathrm{CoFeB}(4) / \mathrm{Mg}(0.5) / \mathrm{MgO}(0.5) / \mathrm{CoFeB}$ (4)/Ru(0.9)/CoFe(3)/MnIr(7.5)/Ta(3)/Ru(7)/capping-layer (thickness in nanometers) was prepared using the dc 
Table 1. List of spacing between the patterned contacts.

\begin{tabular}{cccccccccccc}
\hline \hline Class & \multicolumn{10}{c}{ Spacing between 12 contacts (unit: $\mu \mathrm{m})$} \\
\hline Narrow & 4.5 & 3 & 1.5 & 1.5 & 1.5 & 1.5 & 1.75 & 2.0 & 2.25 & 2.5 & 2.75 \\
Standard & 9.6 & 9.3 & 4.5 & 3 & 1.5 & 1.5 & 1.5 & 1.5 & 5 & 5.7 & 12.4 \\
Wide & 60 & 24 & 15 & 6 & 3 & 3 & 3 & 3 & 9 & 12 & 39 \\
\hline
\end{tabular}

magnetron sputtering method. The sample has the socalled top-pinned junction architecture. The second sample has a similar composition to Sample 1; however, unlike Sample 1, Sample 2 is a bottom-pinned junction of the structure $\mathrm{SiO}_{2} / \mathrm{Ta}(5) / \mathrm{Ru}(40) / \mathrm{MnIr}(7.5) / \mathrm{CoFe}(2) / \mathrm{CoFeB}(1)$ $/ \mathrm{Ru}(0.9) / \mathrm{CoFeB}(4) / \mathrm{Mg}(0.5) / \mathrm{MgO}(0.5) / \mathrm{CoFeB}(4) / \mathrm{Ta}(5) /$ $\mathrm{Ru}(7) /$ capping-layer. Following deposition, the samples were coated with a $\mathrm{SiO}_{2}$ layer $60 \mathrm{~nm}$ thick on the top surfaces. The $\mathrm{SiO}_{2}$ surface was observed by AFM to ensure that no pinholes existed. The probe pads for macroscopic four-point probe measurement were deposited by evaporation on top of the $\mathrm{SiO}_{2}$ layer and were patterned using photolithography. Finally, the Au point contacts, sensing leads to probe pads, and via to MTJ surface for CIPT measurement were defined using e-beam lithography and patterned using reactive ion etch (RIE). The diameter of the contacts was $1.5 \mu \mathrm{m}$ and the spacings between the contacts varied from 1.5 to $60 \mu \mathrm{m}$ and were grouped into three different classes as listed in Table 1. Fig. 1 shows the image of the developed pattern of the point contacts and probes. The magnetic properties of MTJ structures

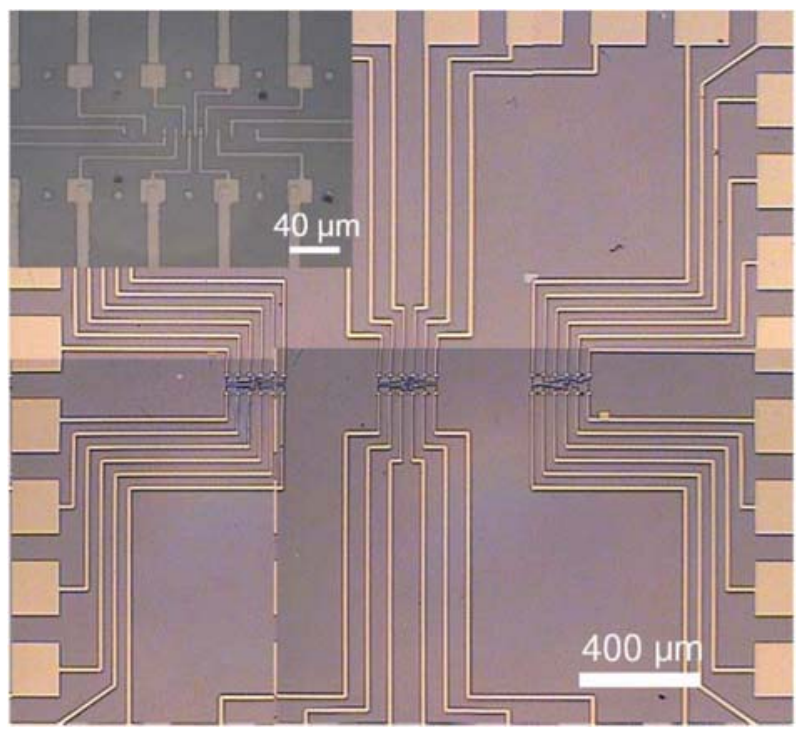

Fig. 1. Image of the MTJ sample with a series of point contacts and probe pads on top surface. The contacts are grouped into three classes according to mean probe spacing: narrow, standard, and wide (from left to right), respectively. Inset (upper left): the magnified SEM image of the sample showing the point contacts and connected sensing leads. were measured using an alternating gradient magnetometer (AGM). The R-H loops of various contact spacings were measured using the four-point probe method with a DC current ranging from 1 to $5 \mathrm{~mA}$. The measurement was repeated and averaged five times for each contact spacing. Magnetoresistance ratios (MR) and RA values were obtained by fitting the measured data to the CIPT model [3].

\section{Results and Discussion}

Fig. 2 shows examples of R-H measurement results for Sample 1 at different probe spacings. Most results have clear resistance change with applied magnetic fields, as shown in Figs. 2(a) and 2(b). However, a few measurements result in highly noisy resistance variations. The situation usually occurred when the contact spacing between $\mathrm{V}^{+}$and $\mathrm{V}^{-}$was much narrower than the spacing between $\mathrm{I}^{+}$and $\mathrm{I}^{-}$as shown in Fig. 2(c). Under such configuration, the voltage difference between two voltage probes was too small and, thus, exhibited a bad signal-tonoise ratio (the background noise was approximately 3 $\mu \mathrm{V}$ in this experiment.). Moreover, similar to situations when using smaller probe spacings in the CIPT microprobe measurement [4], possible positional errors in the patterned $\mathrm{Au}$ contacts could also lead to noisy data at smaller contact spacings.

From measured R-H loops, $\mathrm{R}_{\text {high }}, \mathrm{R}_{\text {low }}$, and $\mathrm{MR}_{\text {cip }}$ (defined by $\left.\left(\mathrm{R}_{\text {high }}-\mathrm{R}_{\text {low }}\right) / \mathrm{R}_{\text {low }}\right)$ can be obtained. The measured $\mathrm{R}_{\text {low }}$ and $\mathrm{MR}_{\text {cip }}$ were fitted to the theoretical expression of Eq. (2) in Ref. [3] by adjusting the four parameters $R_{T}$, $R_{B}, R A$, and MR. Fig. 3 shows the measured and fitted data for top-pinned Sample 1. From the figure, we can see that the discrepancy between measured and fitted data is somewhat larger than the reported results using microprobes [3]. This may arise from the fact that the deposited contact size is much larger than the CIPT microprobe, leading to the shunting of the current. However, the fit results in $\mathrm{MR}=120 \%$ and $\mathrm{RA}=13.65 \Omega-\mu \mathrm{m}^{2}$, which are highly close to the results measured by commercial CIPT tool: $\mathrm{MR}=122 \%, \mathrm{RA}=16.1 \Omega-\mu \mathrm{m}^{2}$.

For the bottom-pinned junction structure, the fit of measured data for Sample 2 results in $\mathrm{MR}=43 \%$ and $\mathrm{RA}=15.89 \Omega-\mu \mathrm{m}^{2}$ as shown in Fig. 4. Note that in this 


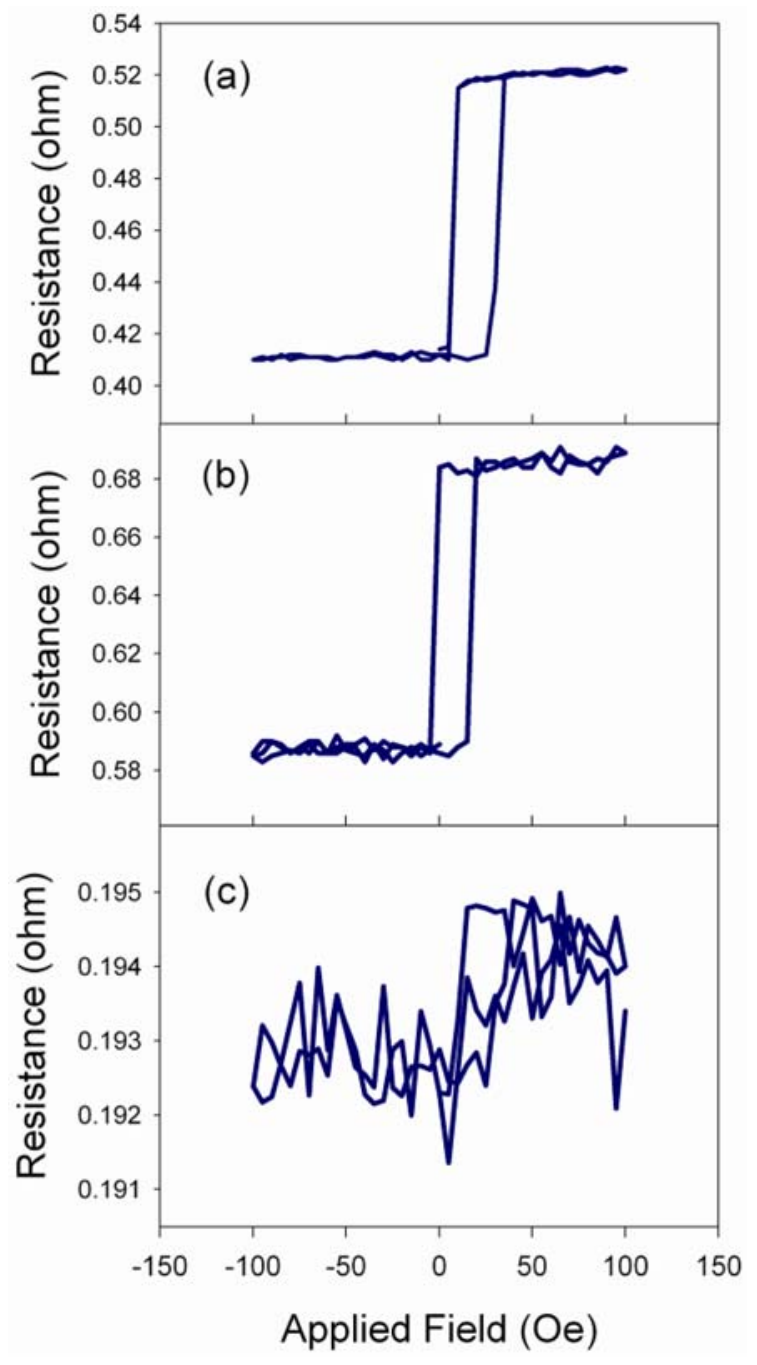

Fig. 2. The R-H loops measured with various probe spacings. The combinations of space between four probes (in units of $\mathrm{mm}$ ) are: (a) 1.5, 1.5, 1.5; (b) 9, 3, 3; and (c) 7.5, 1.5, 3, respectively.

case the sheet resistance of the bottom electrode $R_{B}(5.86$ $\Omega / \square)$ is close the value of the top electrode $R_{T}$ (6.89 $\Omega / \square)$. The spacing for the maximal value of $\mathrm{MR}_{\text {cip }}$ is below the lowest spacing of the contacts and, thus, the $\mathrm{MR}_{\text {cip }}$ decreased with the probe spacing monotonously as expected. The fact that the bottom-pinned MTJ had a smaller MR ratio may originate from the rougher interface induced by the MnIr layer [5,6]. The measurement using the commercial CIPT tool for the same sample results in a higher MR ratio of $51 \%$ and a similar RA value of $16.2 \Omega-\mu \mathrm{m}^{2}$. The results comparisons between the patterned contacts and the standard CIPT method are listed in Table 2. From the table, we can see that using the current-in-plane tunneling measurement through patterned contacts yields close results to those using the commercial

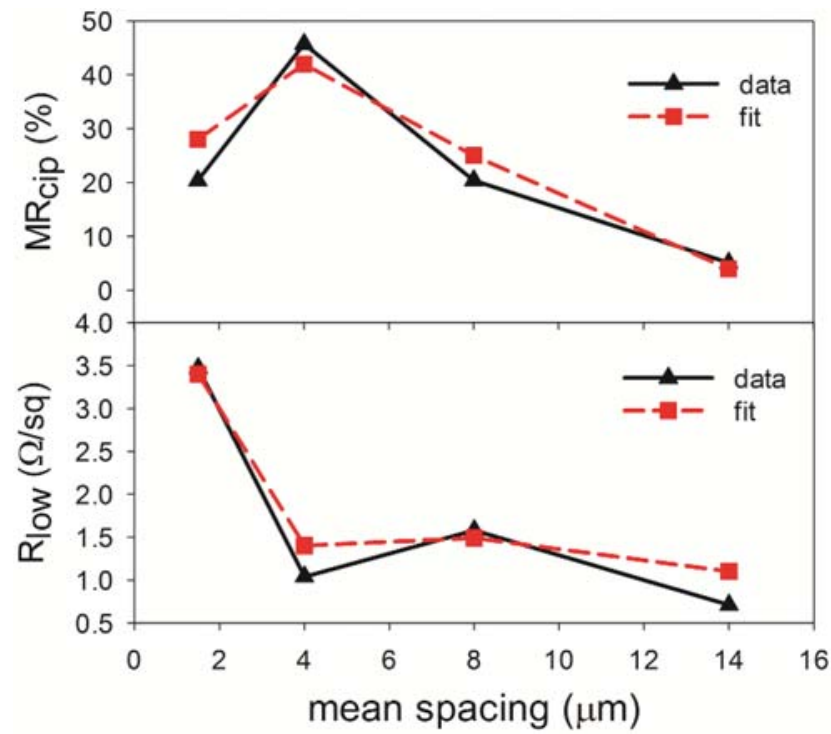

Fig. 3. Comparison of measured data (solid line) with the theoretical fit (dashed line) for the top-pinned MTJ as a function of mean probe spacing. The fit gives $R_{T}=7.3 \Omega / \square, R_{B}=1.35$ $\Omega / \square, \mathrm{RA}=13.65 \Omega-\mu \mathrm{m}^{2}$, and $\mathrm{MR}=120 \%$.

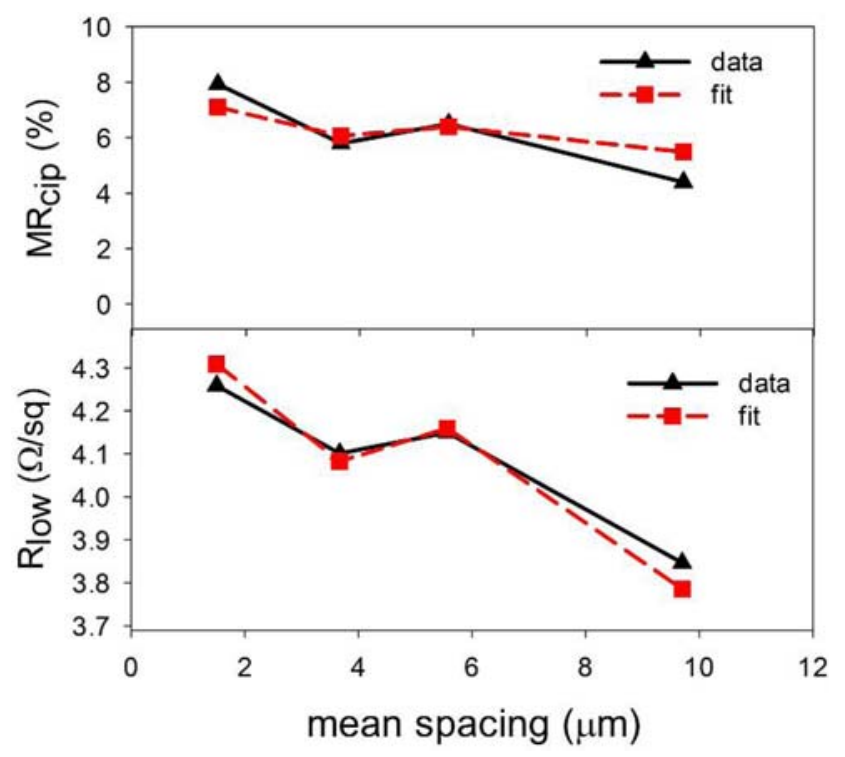

Fig. 4. Comparison of measured data (solid line) with the theoretical fit (dashed line) for the bottom-pinned MTJ as a function of mean probe spacing. The fit gives $R_{T}=6.89 \Omega / \square, R_{B}$ $=5.86 \Omega / \square, \mathrm{RA}=15.89 \Omega-\mu \mathrm{m}^{2}$, and $\mathrm{MR}=43 \%$.

CIPT tool for both top and bottom-pinned junction structures.

\section{Conclusions}

In summary, an alternative CIPT method including deposition of a series of point contacts on the top surface of 
Table 2. Comparison of measurement results from the patterned contacts and commercial CIPT tools.

\begin{tabular}{llcc}
\hline \hline & & Sample 1 & Sample 2 \\
\hline \multirow{2}{*}{ RA $\left(\Omega-\mu \mathrm{m}^{2}\right)$} & patterned contacts & 13.65 & 15.89 \\
& commercial CIPT tool & 16.1 & 16.2 \\
\hline \multirow{2}{*}{ MR (\%) } & patterned contacts & 120 & 43 \\
& commercial CIPT tool & 122 & 51 \\
\hline
\end{tabular}

MTJ films and conventional four-point probe measurement is proposed. Although evaluating speed is slower than the dedicated commercial CIPT tool, the proposed method involves fewer microfabrication steps and is still much faster than conventional microfabrication processes for submicron-sized devices.

\section{Acknowledgment}

This work was supported by the National Science Council, the Republic of China, under Contract No. 97-2112-M224-001, NSC 98-2112-M-224-002-MY3, and by the
Department of Industrial Technology, Ministry of Economic Affairs, the Republic of China, under Contract No. 98EC-17-A-01-S1-026.

\section{References}

[1] S. S. P. Parkin, C. Kaiser, A. Panchula, P. M. Rice, B. Hughes, M. Samant, and S.-H. Yang, Nat. Mater. 3, 862 (2004); Yuasa, T. Nagahama, A. Fukushima, Y. Suzuki, and K. Ando, Nat. Mater. 3, 868 (2004).

[2] S. Isogami, M. Tsunoda, K. Komagaki, K. Sunaga, Y. Uehara, M. Sato, T. Miyajima, and M. Takahashi, Appl. Phys. Lett. 93, 192109 (2008).

[3] D. C. Worledge and P. L. Trouilloud, Appl. Phys. Lett. 83, 84 (2003).

[4] D. W. Abraham, P. L. Trouilloud, and D. C. Worledge, IBM J. Res. \& Dev. 50, 55 (2006).

[5] J. Y. Huang and J. R. Rhee, Materials Science 21, 47 (2003).

[6] C. Y. You, H. S. Goripati, T. Furubayashi, Y. K. Takahashi, and K. Hono, Appl. Phys. Lett. 93, 012501 (2008). 